Perinatology pISSN 2508-4887 • elSSN 2508-4895
Original article

Perinatology Vol. 31, No. 4, December, 2020

https://doi.org/10.14734/PN.2020.31.4.166

\title{
Correlation between Capillary Blood-spotted Filter Paper Thyrotropin Results and Serum Thyroid FunctionTests in Premature Neonates
}

\author{
Moon Bae Ahn, MD, \\ Shin Hee Kim, MD, \\ Won Kyoung Cho, MD, PhD, \\ Kyoung Soon Cho, MD, \\ So-Young Kim, MD, PhD, \\ Byung-Kyu Suh, MD, PhD, \\ Min Ho Jung, MD, PhD
}

Department of Pediatrics, College of Medicine, The Catholic University of Korea, Seoul, Korea

Received: 21 April 2020

Revised: 25 June 2020

Accepted: 5 July 2020

Correspondence to

Min Ho Jung, MD, PhD

Division of Endocrinology,

Department of Pediatrics, College of Medicine, The Catholic University of

Korea, 222 Banpo-daero, Seocho-gu, Seoul 06591, Korea

Tel: +82-2-2258-6756

Fax: +82-2-537-4544

E-mail:jmhpe@catholic.ac.kr

Copyright@ 2020 by The Korean Society of Perinatology

This is an Open Access article distributed under the terms of the Creative Commons Attribution Non-Commercial License (http://creativecommons.org/ license/by-nc/4.0/), which permits unrestricted non-commercial use, distribution, and reproduction in any medium, provided that the original work is properly cited.
Objective: The interpretation of thyroid function test results in preterm infants is affected by various conditions. Neither a definite thyrotropin cut-off nor intercurrent conditions have been clearly determined for premature neonates. This study aimed to determine the specific considerations required in the interpretation of capillary blood thyrotropin concentration by comparing it to serum thyrotropin when screening for congenital hypothyroidism in preterm neonates.

Methods: A retrospective review was performed on 57 premature newborns who were screened for thyroid function test. The comparison of thyrotropin measured by two different methods, dried capillary blood-spotted filter paper using an immunofluorometric assay and serum radioimmunoassay, was performed. Clinical and biochemical factors affecting the difference between the two methods were investigated.

Results: Filter paper thyrotropin concentration showed a positive correlation with the serum thyrotropin concentration. In total, 50.9\% of premature neonates had a difference in the concentration $\geq 3.0 \mathrm{mlU} / \mathrm{L}$ between the methods. In these subjects, the serum thyrotropin concentration was higher, whereas other factors, such as maternal factors, neonatal anthropometric parameters, Apgar score, and corrected age, were not different when compared to that in subjects with a difference of $<3.0 \mathrm{mlU} / \mathrm{L}$.

Conclusion: The filter paper thyrotropin concentration differed from the serum level in a substantial portion of premature neonates, and the degree of deviation may be affected by the serum thyrotropin concentration and gestational age at birth. Further investigations and longitudinal studies are warranted to establish clinical consensus guidelines for hypothyroidism screening in preterm neonates.

Key Words: Congenital hypothyroidism, Dried blood spot testing, Premature birth, Thyrotropin

\section{Introduction}

Congenital hypothyroidism $(\mathrm{CH})$ is one of the common preventable causes of mental retardation, which results in diverse neuropsychiatric deficits. Worldwide screening programs for $\mathrm{CH}$ have contributed to the successful early detection and treatment of $\mathrm{CH}$ over the last 30 years, and the prevalence of $\mathrm{CH}$ has increased 3- to 4-fold when compared to that in the pre- screening era. The European guideline states that $\mathrm{CH}$ screening is highly recommended, particularly in preterm neonates born before 37 weeks of gestation, with a low or very low-birth-weight, and any ill newborns admitted to the neonatal intensive care unit. ${ }^{1}$

In Korea, measurements of thyroid-stimulating hormone (TSH, thyrotropin) using the dried capillary blood-spotted filter paper (FPTSH) method were first introduced in the late 1980s and launched as 1 of the 6 selected items for national newborn screening along with phenylketonuria, galactosemia, maple syrup urine disease, homocystinuria, and congenital adrenal hyperplasia. ${ }^{2.3}$ Measuring TSH using an immunofluorometric assay (IFMA) has gained 
popularity over the use of a radioimmunoassay (RIA) because of its high sensitivity, efficiency, and cost-effectiveness, with no use of a radioactive label. ${ }^{4}$

The interpretation of thyroid function test results in preterm infants is easily affected by various conditions, including an immature hypothalamo-pituitary-thyroid axis, intercurrent illness, or drugs affecting neonatal thyroid function. ${ }^{5}$ However, neither a definite TSH cut-off nor intercurrent conditions have been clearly determined for premature neonates. Both the maternal condition and complications of prematurity could affect the TSH level. Therefore, a method to interpret the TSH value, whether it is measured by serum or FPTSH, remains unclear in the setting of prematurity. Therefore, we aimed to identify any potential factors that should be considered when determining the normal thyroid function in preterm neonates.

\section{Methods}

\section{Study participants}

This retrospective, single-center study initially screened 81 premature neonates born before 37 weeks of gestational. Every enrolled neonate underwent a serum TSH (sTSH) and FPTSH measurement. Twenty-four neonates either had more than 7 days between the 2 measurements or died; thus, they had to be excluded from the initial recruitment. Ultimately, 57 premature newborns were included in this study. Z-scores for birth weight, length, and head circumference were calculated based on the Fenton growth chart. ${ }^{6}$ The presence of maternal factors and co-morbidities associated with prematurity such as respiratory distress syndrome (RDS), bronchopulmonary dysplasia (BPD), intraventricular hemorrhage (IVH), adrenal insufficiency (AI), patent ductus arteriosus (PDA) and necrotizing enterocolitis (NEC) were recorded.

\section{Specimen collection}

Blood draw for serum free thyroxine (fT4), triiodothyronine (T3), and sTSH was performed at any time closest to 48 hours after birth whereas had to be postponed until the critical conditions were resolved. Then, FTPSH was measured either on the day of serum blood test or the date as near as possible. Age and corrected age at each blood collection was recorded.

\section{TSH assays}

From the dried specimens of blood taken from neonates' heels, the FPTSH measurement was obtained by using AutoDELFIA ${ }^{\circledR}$, a second-generation neonatal hTSH assay (human TSH time resolved IFMA, PerkinElmer, Waltham, MA, USA). On the basis of the American Academy of Pediatrics guideline of 1993, the manufacturer's reference intervals for dried blood specimens of normal, borderline, and hypothyroid subjects were $<9.0 \mathrm{mU} / \mathrm{L}$, 9.0-18.0 mU/L, and >18.0 mU/L, respectively. ${ }^{7}$ The manufacturer's inter-assay coefficient of variation was between $6.7 \%$ and 9.0\%. The sTSH specimen was measured using the TSH IRMA $\mathrm{KIT}^{\circledR}$ (in vitro immunoradiometric assay, Beckman Coulter, Brea, CA, USA), whereas fT4 and T3 were measured using the RIA $\mathrm{KIT}^{\circledast}$ (in vitro RIA, Beckman Coulter).

\section{Definitions}

The difference between the FPTSH measurement and sTSH concentration was expressed as $\Delta \mathrm{TSH}$. Potential hypothyroidism was defined when the sTSH value was greater than $6.5 \mathrm{mU} / \mathrm{L}$ or when the fT4 value became less than $0.8 \mathrm{ng} / \mathrm{dL}{ }^{8}$ Overt hypothyroidism for immediate treatment initiation was defined when the sTSH value was greater than $20.0 \mathrm{mU} / \mathrm{L} .{ }^{1}$

\section{Statistical analysis}

The statistical analysis included a descriptive analysis of all the variables, and the results were expressed as the mean \pm standard deviation or the mean, if not normally distributed. Correlations between the FPTSH measurement and sTSH concentration were assessed using the Pearson correlation coefficient. Additional analyses, including the independent $t$-test, chi-square test, and multiple regression were performed to identify any clinical factors affecting $\Delta \mathrm{TSH}$. All statistical analyses were performed using SPSS, version 20.0 (IBM Corp., Armonk, NY, USA).

\section{Ethics statement}

The present study protocol was reviewed and approved by the Catholic Medical Center Institutional Review Board (Approval No. SC16RISI0016). The need for informed consent was waived because of the retrospective study design. 


\section{Results}

\section{Demographic and baseline characteristics}

Demographic and baseline characteristics of the participants are shown in Table 1 . The study sample was evenly distributed by sex, with $31(54.4 \%)$ male premature neonates and 26 $(45.6 \%)$ female premature neonates (with a mean gestational age [GA] of $33.6 \pm 2.7$ weeks). Overall, $78.9 \%$ of neonates were born with a normal birth weight for GA, and $75.4 \%$ of the deliveries were by cesarean section. Sixteen (28.1\%) mothers experienced premature rupture of the membranes, 8 (14.0\%) had pre-eclampsia, 7 (12.2\%) had gestational diabetes mellitus, and $4(7.0 \%)$ had perinatal infection. Among the comorbid conditions that were noted, respiratory complications were predominant (24 neonates had RDS (42.1\%) and 9 had BPD (15.7\%) of either mild, moderate, severe degree), followed by IVH (22.8\%) grading between I and IV (Papile), AI (14.0\%), PDA (7.0\%), and NEC (3.5\%) staging between I and III (Modified Bell). ${ }^{9,10}$ All neonates with PDA were symptomatic and screened by echocardiography at 4 days after birth.

Table 1. Demographic and Baseline Characteristics of the Study Participants

\begin{tabular}{lc}
\hline Characteristic & Value \\
\hline Sex (male) & $31(54.4)$ \\
GA (weeks) & $33.6 \pm 2.7(26.0-36.7)$ \\
\hline BW for GA & $45(78.9)$ \\
AGA & $9(15.8)$ \\
SGA & $3(0.5)$ \\
LGA & $14(24.6)$ \\
Methods of delivery & $43(75.4)$ \\
Vaginal & \\
Cesarean section & \\
Apgar score & $5.9 \pm 1.8(1.0-9.0)$ \\
1 minute & $7.6 \pm 1.4(3.0-10.0)$ \\
5 minutes & $-0.3 \pm 0.9(-2.2-2.0)$ \\
BW-SDS* & $0.5 \pm 1.2(-3.3-2.8)$ \\
BL-SDS & $0.0 \pm 1.1(-2.7-2.4)$ \\
\hline BHC-SDS
\end{tabular}

All values are expressed as mean \pm standard deviation or number (\%). Values in parentheses represent the range (minimum-maximum) if not written in percentage.

Abbreviations: GA, gestational age; BW, birth weight; AGA, appropriate for gestational age; SGA, small for gestational age; LGA, large for gestational age; SDS, standard deviation score; BL, birth length; BHC, birth head circumference.

${ }^{*}$ The calculation was based on the Fenton growth chart. ${ }^{6}$

\section{Descriptive data on thyroid hormone screening}

Table 2 describes the mean FPTSH measurements and sTSH concentrations. The mean $\Delta \mathrm{TSH}$ was $3.3 \pm 2.5 \mathrm{mU} / \mathrm{L}$ with a time interval of $3.1 \pm 2.7$ days. Serum T3 and fT4 values were $1.3 \pm 1.4$ $\mathrm{ng} / \mathrm{mL}$ and $1.4 \pm 0.3 \mathrm{ng} / \mathrm{dL}$ (with a mean age at collection of 13.7 \pm 10.2 days, corrected age: $35.6 \pm 1.8$ weeks), respectively. Furthermore, 30 neonates (52.6\%) were potentially in a hypothyroid state. Only 1 neonate (1.7\%) was diagnosed with $\mathrm{CH}$ and required thyroxine replacement.

\section{Statistical association between the FPTSH measurement and}

\section{sTSH concentration}

In the correlation analysis, the FPTSH measurement showed a linear relationship with the sTSH concentration $\left(r^{2}=0.589\right.$, $P<0.001$ ) (Fig. 1). The proportions of neonates with a $\Delta$ TSH less

Table 2. Thyroid Hormone Concentration and Age Recorded

\begin{tabular}{llcc}
\hline & \multicolumn{1}{c}{ Value $^{*}$} & \multicolumn{1}{c}{ Age (days) } & Corrected age (weeks) \\
\hline FPTSH & $2.7 \pm 1.9(0.4-9.1)$ & $12.9 \pm 8.8(5.0-44.0)$ & $35.5 \pm 1.9(30.0-39.2)$ \\
sTSH & $5.8 \pm 4.0(0.4-9.6)$ & & \\
T3 & $1.3 \pm 1.4(0.3-11.1)$ & $13.7 \pm 10.2(5.0-44.0)$ & $35.6 \pm 1.8(30.5-38.5)$ \\
fT4 & $1.4 \pm 0.3(0.7-2.3)$ & & \\
$\Delta$ TSH & $3.3 \pm 2.5(0.1-10.6)$ & & \\
\hline
\end{tabular}

All values are expressed as mean \pm standard deviation. Values in parentheses represent the range (minimum-maximum).

Abbreviations: FPTSH, dried capillary blood-spotted filter paper thyrotropin; STSH, serum thyroid-stimulating hormone; T3, triiodothyronine; fT4, free thyroxine; TSH, thyroid-stimulating hormone.

*Units of measure are $\mathrm{mU} / \mathrm{L}$ for TSH, ng/mL for T3, and ng/dL for fT4.

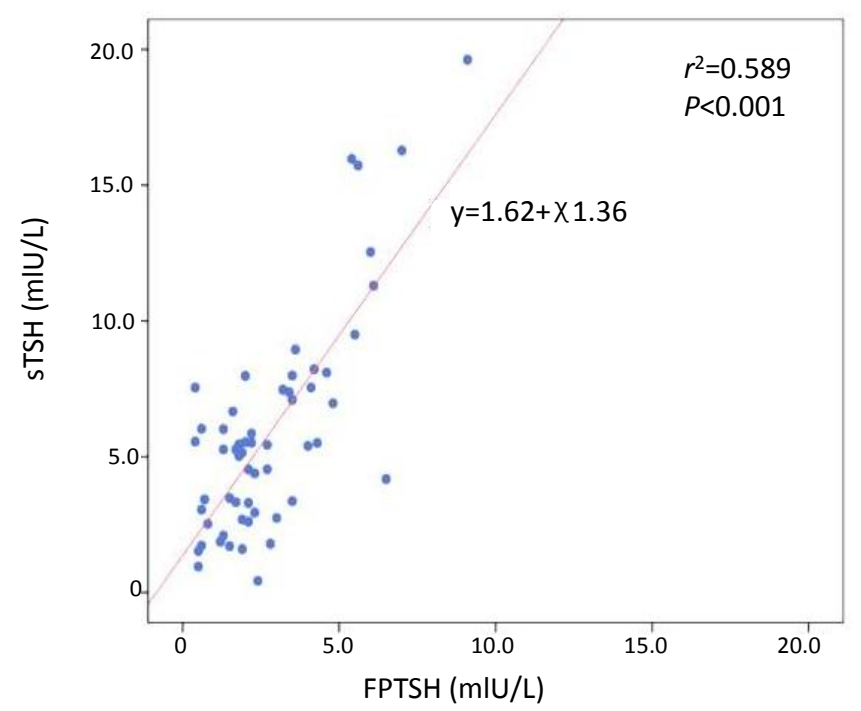

Fig. 1. Correlation analysis of capillary blood filter paper thyrotropin (FPTSH) with serum thyroid-stimulating hormone (sTSH). 
than $1.0,2.0,3.0$, and 4.0 were $17 \%, 36 \%, 49 \%$, and $66 \%$, respectively (Fig. 2). The entire population was subdivided into 2 groups by a $\triangle \mathrm{TSH}$ of 3.0 based on the sample size with an even distribution. The FPTSH measurement $(P=0.021)$ and sTSH concentration $(P<0.001)$ were higher in the group with a $\Delta \mathrm{TSH}$ $\geq 3.0$ when compared to that in the group with a $\Delta \mathrm{TSH}<3.0$. Other factors, such as GA or birth anthropometric data, showed no significant association. Multiple regression analysis was performed to investigate the factors that increased the extent of $\triangle \mathrm{TSH}$, and the sTSH concentration was positively correlated with $\Delta \mathrm{TSH}(\mathrm{B}=0.60, P<0.001$ ), whereas GA was inversely related to $\Delta \mathrm{TSH}(\mathrm{B}=-0.23, P=0.022)$ (Table 3 ).

\section{Discussion}

Our findings suggest that TSH, measured by filter paper, has

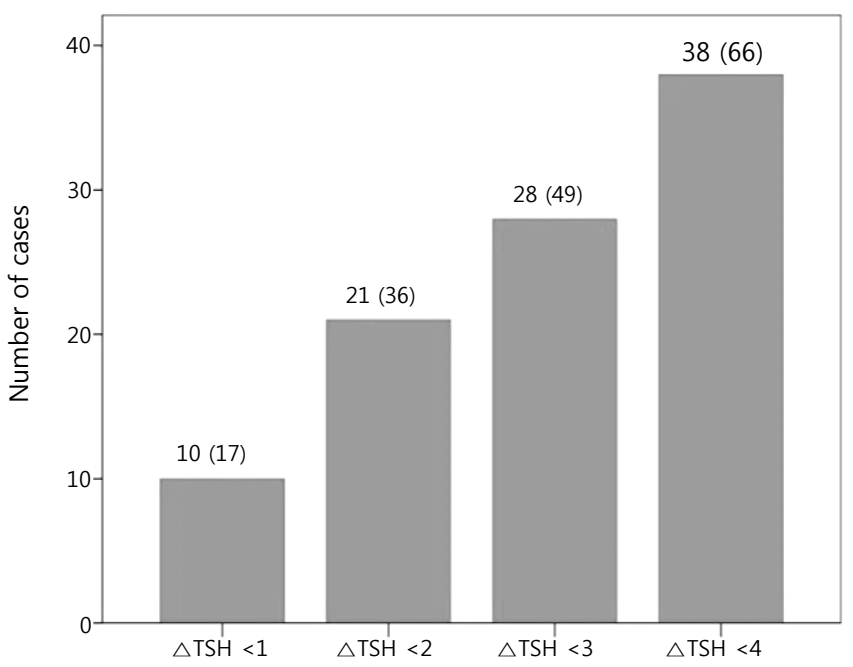

Fig. 2. Difference between filter paper and serum thyroid-stimulating hormone (sTSH) (mlU/L).

Table 3. Multivariate Logistic Regression for Differences in the Thyrotropin Level Between the Dried Capillary Blood-spotted Filter Paper Measurement and Serum Measurement

\begin{tabular}{lccc}
\hline & Odds ratio & $95 \% \mathrm{Cl}$ & $P$-value \\
\hline GA (weeks) & -0.23 & $-0.45 \sim-0.02$ & 0.022 \\
STSH (mU/L) & 0.60 & $0.53-0.68$ & $<0.001$ \\
BW-SDS & -0.02 & $-0.55-0.50$ & 0.928 \\
BL-SDS & 0.10 & $-0.25-0.45$ & 0.573 \\
\hline
\end{tabular}

Abbreviations: $\mathrm{Cl}$, confidence interval; $\mathrm{GA}$, gestational age; $\mathrm{TSH}$, thyroid-stimulating hormone BW, birth weight; SDS, standard deviation score; BL, birth length.

*Hosmer Lemeshow Goodness-of-fit test. a significant correlation with the serum level. Nonetheless, TSH measured by dried capillary blood-spotted filter paper within 2 weeks after birth was remarkably low compared to the serum concentration. About half of the total patients had a $\Delta \mathrm{TSH} \geq 3.0$ $\mathrm{mU} / \mathrm{L}$. This difference in TSH had a positive association with the sTSH concentration, whereas it had an inverse relationship with GA.

Abnormal thyroid function is one of the most common endocrinopathies among premature neonates. In prematurity, TSH-releasing hormone secretion and the thyroid-gland response to TSH are compromised, and iodination along with the conversion of thyroxine to T3 activity is reduced. ${ }^{11}$ Each of these events combined with comorbidities associated with the prematurity including RDS, BPD, IVH, AI, PDA and NEC contribute to the immaturity of the hypothalamo-pituitary-thyroid axis. Thyroid gland reserve (iodine and thyroglobulin stores) is limited, and predisposition to nonthyroidal illness syndrome is higher in term infants than in premature infants. ${ }^{12}$ The more premature the baby, the higher the chance of TSH escaping the boundaries.

Since the IFMA was introduced in the 1980s as an alternative to the RIA, its sensitivity and reliability have been upgraded. ${ }^{11}$ With an advantage of non-radioactive labels, measuring the thyroid hormone level using the IFMA has shown a potential to yield analogous performance characteristics compared to the RIA. ${ }^{12}$ Second or third generation assays are routinely used in most clinical settings, and a fourth-generation assay with superior sensitivity is currently available. It took approximately 20 years until the FPTSH method was fully verified and implemented in the national newborn screening program in Korea. The screening strategy for $\mathrm{CH}$ in Korea is to measure the primary TSH level, and TSH screening is included as 1 of the 6 selected inborn errors of metabolism diseases determined by the Korean Centers for Disease Control and Prevention. Therefore, the cost of this screening is covered by the National Health Insurance Corporation.

As earlier studies have mentioned, dried capillary filter paper is a universal method in newborn screening programs in many countries. $^{4,13}$ The less invasive approach and simplified procedure are strong benefits in the very low-birth-weight population. Nevertheless, an unsatisfactory difference in the TSH concentration has always existed between the filter paper measurement and serum values. The cut-off for initiation of treat- 
ment is controversial and depends on the interpretation of the serum TSH value in the clinical setting. However, FPTSH might not always be a reliable indicator in acutely ill premature neonates. Although there are many published reference intervals of thyroid function in preterm neonates, no consensus has been established yet, and the reliability of the cut-off for FPTSH in this population is not accredited. ${ }^{14,15}$

Overall, $61.2 \%$ of our study population had periconceptual factors, and $91.1 \%$ had prematurity-associated comorbid conditions. GA was an inversely associated factor against the TSH difference. Our results are consistent with prior knowledge that neonates born with an extremely low-birth-weight or a short gestation are prone to developing serious complications, and such conditions as well as periconceptual factors could affect the diagnosis of hypothyroidism by under or overestimating the TSH level. ${ }^{16,17}$ In addition, extremely low-birth-weight infants tend to have a negative iodine balance and might be more susceptible to iodine exposure. ${ }^{18}$ Consequently, the question becomes inevitable whether the current FPTSH cut-off for normal healthy babies should be applied to premature newborns. A TSHbased screening strategy is challenged to detect $\mathrm{CH}$ of central origin, and a false-positive TSH measurement complicates its diagnosis even more among premature neonates. ${ }^{19}$ Furthermore, our result raises the possibility of overlooked elevation in the TSH level even when the FPTSH measurement is within normal range. The FPTSH measurement alone cannot validate the presence of serum TSH elevation or a diagnosis of hypothyroidism. Measurements of TSH obtained by dried blood spots might be unreliable because of aberrant protein binding and decreased thyroxine-binding globulin observed in sick and premature children. ${ }^{20,21}$ Although interpreting thyroid function tests is challenging and diagnostic tools are limited, we suggest evaluating thyroxine and TSH using the dried capillary blood-spotted filter paper, especially in premature infants. Further, any abnormal findings should be verified based on serum concentrations.

This study has a few critical limitations. It was a retrospective, single-center study based on a small number of neonates receiving critical care, and only 1 neonate was diagnosed with hypothyroidism. Increasing our sample size might have increased the power to detect factors that affect the $\Delta \mathrm{TSH}$. As we only investigated the presence of maternal and prematurity-associated comorbid conditions, we did not consider that the severity might have affected the results. This study also did not propose any appropriate cut-off value for the FPTSH measurement. However, our results support the idea that any given cut-off for the FPTSH measurement cannot guarantee definite hypothyroidism or avoid measurement of the sTSH concentration. The correlation of TSH measurements for screening of thyroid disorders, whether by blood-spotted filter paper or serum specimens, relies upon the individual's health condition and comorbid illness, especially among preterm newborns.

In conclusion, the FPTSH level differed from the serum level in a substantial portion of premature neonates, and the degree of deviation may be affected by the sTSH level and GA at birth. Various conditions, including the complications in premature neonates, interfere with the correct interpretation of thyroid function. To avoid overlooking hypothyroidism, a diagnostic approach should be described before measuring the serum thyroxine and TSH concentrations. Further investigations and longitudinal studies are warranted to establish clinical consensus guidelines for hypothyroidism screening in preterm neonates.

\section{Acknowledgement}

This research was not supported by any funding.

\section{Conflict of interest}

No potential conflict of interest relevant to this article was reported.

\section{References}

1) Léger J, Olivieri A, Donaldson M, Torresani T, Krude H, van Vliet G, et al. European Society for Paediatric Endocrinology consensus guidelines on screening, diagnosis, and management of congenital hypothyroidism. Horm Res Paediatr 2014;81:80-103.

2) Lee $\mathrm{DH}$. Newborn screening of inherited metabolic disease in Korea. Korean J Pediatr 2006;49:1125-39.

3) Song JY, Son DW, Kim Bl, Yang SW, Choi JH, Yoon CK, et al. Re-evaluation of TSH screening TEST in neonates. Korean J Pediatr 1993;36:1502-6.

4) Rustama DS, Rosilawati E, Permatagalih V. Neonatal screening for congenital hypothyroidism: primary thryotropin screening: comparison of 
false positive rate using radioimmunossay (RIA) Vs fluorometric assay (FIA). Int J Pediatr Endocrinol 2013 Oct 3 [Epub]. https://doi.org/10.1186 /1687-9856-2013-S1-P151.

5) Hallett A, Evans C, Moat S, Barton J, Warner J, Gregory JW. Hypothyroidism in preterm infants following normal screening. Ann Clin Biochem 2011; 48:572-4.

6) Fenton TR, Kim JH. A systematic review and meta-analysis to revise the Fenton growth chart for preterm infants. BMC Pediatr 2013;13:59.

7) American Academy of Pediatrics. AAP Section on Endocrinology and Committee on Genetics, and American Thyroid Association Committee on Public Health: newborn screening for congenital hypothyroidism: recommended guidelines. Pediatrics 1993;91:1203-9.

8) Sperling MA. Pediatric Endocrinology, 4th ed. Philadelphia: Elsevier; 2014.

9) Gregory KE, Deforge CE, Natale KM, Phillips M, Van Marter LJ. Necrotizing enterocolitis in the premature infant: neonatal nursing assessment, disease pathogenesis, and clinical presentation. Adv Neonatal Care 2011;11:155-64; quiz 65-6.

10) Kuban K, Teele RL. Rationale for grading intracranial hemorrhage in premature infants. Pediatrics 1984;74:358-63.

11) Jung MH. Endocrine disorders in the sick and preterm newborn. J Korean Soc Pediatr Endocrinol 2011;16:73-9.

12) Fisher DA. Thyroid system immaturities in very low birth weight premature infants. Semin Perinatol 2008;32:387-97.

13) Zhou Y, Xia X, Xu Y, Ke W, Yang W, Li Q. Application of europium(III) chelates-bonded silica nanoparticle in time-resolved immunofluorometric detection assay for human thyroid stimulating hormone. Anal Chim Acta 2012;722:95-9.
14) Ayyad AH, Hashemipour M, Hovsepian S, Kooshki AM, Afshari M. The relation between serum and filter paper TSH level in neonates with congenital hypothyroidism. Adv Biomed Res 2014;3:23.

15) Christensen-Adad FC, Mendes-Dos-Santos CT, Goto MMF, Sewaybricker LE, D'Souza-Li LFR, Guerra-Junior G, et al. Neonatal screening: 9\% of children with filter paper thyroid-stimulating hormone levels between 5 and $10 \mu \mathrm{lU} / \mathrm{mL}$ have congenital hypothyroidism. J Pediatr (Rio J) 2017 93:649-54

16) Adams LM, Emery JR, Clark SJ, Carlton El, Nelson JC. Reference ranges for newer thyroid function tests in premature infants. J Pediatr 1995;126: 122-7.

17) Williams FL, Simpson J, Delahunty C, Ogston SA, Bongers-Schokking Jנ Murphy $\mathrm{N}$, et al. Developmental trends in cord and postpartum serum thyroid hormones in preterm infants. J Clin Endocrinol Metab 2004;89: 5314-20.

18) Ryckman KK, Spracklen CN, Dagle JM, Murray JC. Maternal factors and complications of preterm birth associated with neonatal thyroid stimu lating hormone. J Pediatr Endocrinol Metab 2014;27:929-38.

19) Korada M, Pearce MS, Avis E, Turner S, Cheetham T. TSH levels in relation to gestation, birth weight and sex. Horm Res 2009;72:120-3.

20) Cherella CE, Wassner AJ. Congenital hypothyroidism: insights into pathogenesis and treatment. Int J Pediatr Endocrinol 2017 Oct 2 [Epub]. https://doi.org/10.1186/s13633-017-0051-0.

21) Larson C, Hermos R, Delaney A, Daley D, Mitchell M. Risk factors associ ated with delayed thyrotropin elevations in congenital hypothyroidism. J Pediatr 2003;143:587-91. 